\title{
Impact of latent tuberculosis infection on health and wellbeing: a systematic review and meta-analysis
}

\author{
Yen Jun Wong (1) ${ }^{1}$, Noorliza Mohd Noordin², Salmaan Keshavjee ${ }^{3}$ and \\ Shaun Wen Huey Lee (10) $1,4,5,6$
}

Affiliations: ${ }^{1}$ School of Pharmacy, Monash University Malaysia, Bandar Sunway, Malaysia. ${ }^{2}$ National Public Health Laboratory, Ministry of Health, Sungai Buloh, Malaysia. ${ }^{3}$ Dept of Global Health and Social Medicine, Harvard Medical School, Harvard University, Boston, MA, USA. ${ }^{4}$ Asian Centre for Evidence Synthesis in Population, Implementation and Clinical Outcomes (PICO), Health and Well-being Cluster, Global Asia in the 21st Century (GA21) Platform, Monash University Malaysia, Subang Jaya, Malaysia. ${ }^{5}$ Gerontechnology Laboratory, Global Asia in the 21st Century (GA21) Platform, Monash University Malaysia, Subang Jaya, Malaysia. ${ }^{6}$ School of Pharmacy, Taylor's University Lakeside Campus, Subang Jaya, Malaysia.

Correspondence: Shaun Wen Huey Lee, School of Pharmacy, Monash University Malaysia, Jalan Lagoon Selatan, 46150 Bandar Sunway, Malaysia. E-mail: shaun.leedmonash.edu

@ERSpublications

The health and wellbeing of individuals with latent tuberculosis infection (LTBI) requires considerable attention to overcome treatment-related challenges and concerns over stigma in an effort to improve LTBI care https://bit.ly/34e3lt

Cite this article as: Wong YJ, Noordin NM, Keshavjee S, et al. Impact of latent tuberculosis infection on health and wellbeing: a systematic review and meta-analysis. Eur Respir Rev 2021; 29: 200260 [https://doi. org/10.1183/16000617.0260-2020].

ABSTRACT The impact of latent tuberculosis infection (LTBI) on health and wellbeing is not well understood. This review aims to evaluate the health and wellbeing of individuals with LTBI.

A systematic literature search was performed to assess studies reporting patient-reported outcomes in LTBI management including health-related quality of life (HRQoL), health utilities, disease burden and experience of individuals with LTBI. A pooled analysis was performed to estimate the effect of LTBI on HRQoL.

A total of 4464 studies were screened, of which 13 eligible articles describing nine unique studies were included for review. The HRQoL of individuals with LTBI and without tuberculosis (TB) infection were comparable, and better than patients with active TB disease. However, individuals with LTBI reported poorer mental health compared with individuals without TB infection (mean difference $-4.16,95 \%$ CI $-7.45--0.87 ; \mathrm{p}=0.01)$. Qualitative studies suggest the presence of fear, anxiety and stigma in individuals with LTBI.

This review highlights potential psychosocial challenges in individuals with LTBI despite the absence of clinical symptoms. While their quality of life was marginally affected, this could be evidence to support LTBI management in preventing TB re-activation and the severe consequences of active TB disease that affect all domains of HRQoL.

\section{Introduction}

Tuberculosis (TB) is one of the top 10 causes of global mortality, with an estimated 1.5 million deaths due to TB in 2018, globally [1]. TB imposes a negative impact on the physical wellness of affected patients due

\section{This article has supplementary material available from err.ersjournals.com}

Provenance: Submitted article, peer reviewed

Received: 8 Aug 2020 | Accepted: 25 Sept 2020

Copyright (CERS 2021. This article is open access and distributed under the terms of the Creative Commons Attribution Non-Commercial Licence 4.0. 
to pulmonary impairment and detrimental clinical symptoms such as fatigue, haemoptysis and significant weight loss [1-3]. In addition to physical symptoms, patients with TB also experience psychosocial pressures, such as stress due to the financial burden of treatment and the possibility of being dismissed from work $[4,5]$.

As part of the World Health Organization's (WHO) End TB Strategy, the United Nation High Level Meeting on TB in 2018 called for efforts to focus on latent tuberculosis infection (LTBI) management as one of the TB preventive care approaches to control and to reduce the incidence of newly diagnosed active TB disease resulting from TB re-activation $[6,7]$. Since individuals with LTBI are clinically asymptomatic, diagnosing and treating them for LTBI could be beneficial in preventing TB re-activation [7, 8]. Through treatment, individuals with LTBI can be protected from the deleterious repercussions of active TB disease including the disease and socioeconomic burden, potentially preserving their health-related quality of life (HRQoL).

However, individuals treated for LTBI may still experience marginal effects on their health and wellbeing because of the treatment side effects as well as the possibility of stigmatisation and discrimination from society for as a result of an LTBI diagnosis [9-12]. The magnitude of impact on the health outcomes and psychosocial facet in the life of individuals with LTBI has not been well understood to date. In contrast, numerous patient-reported outcome (PRO) instruments with different evaluation features have been used to assess the HRQoL and health utilities of patients with active TB disease $[2,3,9,13,14]$.

In patient-centred care practice, PRO measures play an important role in capturing patients' subjective perceptions of health evidence of treatment response, physical symptoms, psychological challenges, quality of life and wellbeing. This helps in addressing patients' unmet needs to improve the quality of care with a valued outcome in a holistic health service $[15,16]$. This review aims: 1) to identify studies that reported the health and wellbeing of individuals diagnosed with LTBI; 2) to evaluate the impact of LTBI on HRQoL compared with patients with active TB disease and individual without TB infection; and 3) to assess the psychometric properties of health outcome instruments. To allow for a more comprehensive understanding on our proposed objectives and outcomes, this review includes literature from participants of all age groups.

\section{Methods}

This systematic review and meta-analysis was conducted according to the Meta-analysis of Observational Studies in Epidemiology (MOOSE) guidelines (table S1) [17]. The protocol of this review was registered in PROSPERO (Registration ID CRD42020148537).

\section{Search strategy}

A systematic search was performed in six electronic databases: PubMed, EMBASE, PsycINFO, CINAHL-Plus, Cochrane Central Register of Controlled Trials (CENTRAL) and ProQuest using a combination of key words and MeSH terms "("latent tuberculosis" OR LTBI OR tuberculosis prevent* OR inactive tuber*) AND ("quality of life" OR "health related quality of life" OR wellbeing OR health status OR disability OR "health outcome" OR "patient reported outcome" OR "quality adjusted life year" OR "disability adjusted life year" OR anxiety OR stress OR depression OR mobility OR self-care OR usual activities OR pain OR discomfort OR physical health OR physical well-being OR physical HRQoL OR physical functioning OR bodily pain OR general health OR vitality OR social functioning OR social health OR social well-being OR social HRQoL OR social relationship OR role emotion OR role physical OR "mental health" OR mental well-being OR mental HRQoL OR stigma OR psychosoc* OR psychological health OR fatigue OR emotional stress OR environmental health OR education OR employment OR income OR illness perception OR treatment perception OR morbidity OR mortality)" from inception to 27 July 2020. This was supplemented with manual searches on the International Journal of Tuberculosis and Lung Disease, CHEST, the European Respiratory Journal and the reference lists of potentially relevant studies. The full list of databases and search terms used is presented in table S2.

\section{Study eligibility}

Studies which evaluated the health and wellbeing in LTBI irrespective of study designs or settings were assessed in the current review. Studies were included if they fulfilled the following eligibility criteria. Included studies: (1) examined individuals diagnosed with LTBI who either tested positive for tuberculin skin test (TST) or interferon-gamma release assay (IGRA) in the absence of clinical symptoms and/or had TB ruled out with chest radiographs and microbiological assessments; (2) assessed health and/or wellbeing as a result of LTBI, including the use of PRO measures using either a generic or disease-specific instrument, stigma scale, subjects' experiences, generic measure of disease burden and mortality risk; and 3) were published in English. We only included literature published in English due to a lack of resources 
in translation proficiency. We excluded case reports, conference abstracts, review articles, economic analysis and letters.

Data extraction and methodological quality assessment

Two reviewers (Y.J. Wong and S.W.H. Lee) independently screened the titles and abstracts of the retrieved articles. Full texts of the identified studies were then reviewed in accordance with the eligible criteria. Any disagreement was discussed and resolved by discussion between the two reviewers. Data from eligible studies were extracted and tabulated using a standardised extraction template. Data extracted include study methods, participants, HRQoL outcome instruments, outcome measurement and relevant remarks (table 1 and table S3). In the event that two studies had reported the same cohort of participants, we extracted the

\begin{tabular}{|c|c|c|c|c|c|c|c|c|}
\hline Study design & Study location & LTBI & TB & $\begin{array}{l}\text { Without TB } \\
\text { infection }\end{array}$ & $\begin{array}{l}\text { Recruitment } \\
\text { duration }\end{array}$ & $\begin{array}{l}\text { Instrument/ } \\
\text { measurement }\end{array}$ & $\begin{array}{l}\text { Reported } \\
\text { health } \\
\text { outcome }\end{array}$ & [Ref.] \\
\hline Longitudinal & $\begin{array}{l}\text { TB clinics, MCI } \\
\text { and JGH, } \\
\text { Canada }\end{array}$ & 105 & 48 & 110 & $\begin{array}{l}\text { June } 2008 \text {-October } \\
2011 \text { (40 months) }\end{array}$ & $\begin{array}{c}\text { 1) SF-36 } \rightarrow \text { SF-6D } \\
\text { utilities } \\
\text { 2) SG }\end{array}$ & $\begin{array}{l}\text { HRQoL [19] } \\
\text { Health } \\
\text { utilities [18] }\end{array}$ & {$[18,19]$} \\
\hline Cross-sectional & $\begin{array}{l}\text { TB clinic, MCI, } \\
\text { Canada }\end{array}$ & 35 & 22 & & $\begin{array}{l}1 \text { September 1999- } \\
30 \text { September } 2000 \\
\text { (13 months) }\end{array}$ & $\begin{array}{l}\text { 1) VAS } \\
\text { 2) } S G \\
\text { 3) } S F-36 \\
\text { 4) } E Q-5 D\end{array}$ & HRQoL & {$[21,22]$} \\
\hline Longitudinal & $\begin{array}{l}\text { TB clinic, British } \\
\text { Columbia } \\
\text { Centre for } \\
\text { Disease Control, } \\
\text { Canada }\end{array}$ & 102 & 104 & & $\begin{array}{l}\text { 2005-2006 } \\
\text { (12 months) }\end{array}$ & $\begin{array}{c}\text { 1) SF-36 } \\
\text { v2 } \rightarrow \text { SF-6D utilities } \\
\text { 2) BDI } \\
\text { 3) HUI2, HUI3 } \\
\text { 4) VAS }\end{array}$ & $\begin{array}{l}\text { HRQoL [24] } \\
\text { Health } \\
\text { utilities [25] }\end{array}$ & {$[24,25]$} \\
\hline Retrospective & $\begin{array}{l}\text { TB registries, } 20 \\
\text { different sites in } \\
\text { the USA }\end{array}$ & 7282 & 3853 & & $\begin{array}{l}\text { 1993-31 December } \\
2002 \text { (9 years) }\end{array}$ & 1) Mortality risk & Mortality & {$[26]$} \\
\hline Retrospective & $\begin{array}{l}\text { TB clinic, TCPH, } \\
\text { Texas, USA }\end{array}$ & 210 & 107 & & $\begin{array}{c}7 \text { July } 2005-7 \\
\text { November } 2006 \\
\text { (16 months) }\end{array}$ & 1) $S G R Q$ & $\begin{array}{l}\text { HRQoL [27] } \\
\text { QALY [22] }\end{array}$ & {$[27,28]$} \\
\hline Cross-sectional & $\begin{array}{l}\text { Dispensary of TB } \\
\text { Kayseri, Turkey }\end{array}$ & 108 & 196 & 196 & $\begin{array}{c}15 \text { September } \\
2003-15 \\
\text { September } 2004 \\
\text { (12 months) }\end{array}$ & $\begin{array}{l}\text { 1) SF-36 } \\
\text { 2) BDI }\end{array}$ & HRQoL & [30] \\
\hline
\end{tabular}

LTBI: latent tuberculosis infection; TB: tuberculosis; MCI: Montreal Chest Institute; JGH: Jewish General Hospital; SF-36: 36-item Short-Form Health Survey; SF-6D: Short-Form Six-Dimension; SG: standard gamble tasks; HRQoL: health-related quality of life; EMIC: Explanatory Model Interview Catalogue; VAS: visual analogue scale; EQ-5D: EuroQol-5 dimensions; TB-OPD: Tuberculosis outpatient department; SF-36 v2: 36-item Short-Form Health Survey version 2; BDI: Beck Depression Inventory; HUI2: Health Utilities Index Mark 2; HUI3: Health Utilities Index Mark 3; TCPH: Tarrant County Public Health; SGRQ: St George's Respiratory Questionnaire; QALY: quality-adjusted life year; RHS-15: Refugee Health Screener-15. 
information from the latest published study. To supplement data collection, we also contacted the corresponding authors of two studies for additional data. Both authors responded but were unable to provide us with the information required $[18,19,24,25]$.

The methodological quality of the included studies was then assessed independently by two reviewers using the Newcastle-Ottawa Scale (NOS). Three criteria of the study design were evaluated, which included the selection of the sample in terms of sample representativeness, comparability between cohorts and outcomes together with follow-up of the subjects from the studies [31]. Qualitative studies were evaluated using the Critical Appraisal Skill Programme (CASP) checklist for qualitative research to assess the study validity, clear statements of findings and the value of research to society [32].

\section{Data analysis}

The primary outcome of this review was stratified into mental health and wellbeing, physical health and wellbeing as well as social health and wellbeing. The secondary outcome of this study was the psychometric properties of the health outcome instruments. We summarised all data narratively in this systematic review. Where appropriate, the effects on the health and wellbeing focusing on HRQoL and health utilities with mean scores available were pooled using DerSimonian and Laird random effects meta-analysis and presented as mean differences (MD) with their corresponding 95\% CIs. Data which were presented using medians and interquartile ranges were converted to means and standard deviations according to the study by WAN and colleagues for meta-analyses [33]. Heterogeneity between studies were assessed using $I^{2}$ statistics. All statistical analyses were performed using Review Manager 5 (RevMan 5, version 5.3).

\section{Results \\ Study characteristics}

The search identified a total of 4464 articles, and a total of 13 articles describing nine unique studies were included in this review [18-30] (figure 1). These studies were conducted from 1999 to 2020 and enrolled a total of 32107 participants, comprising 8056 individuals with LTBI, 4330 with active TB disease, and 19721 individuals without TB infection (see table 1). The cohort size of studies varied from 16 to 19523 participants, and all participants were reported to be at least 16 years old. Eight studies were conducted in countries with a low TB burden (three in Canada, three in the United States and two in Sweden) [18-29], while one study was conducted in Turkey, an intermediate TB burden country [30]. We did not find any studies from a high TB burden country.

Three studies were cross-sectional in design $[21,22,29,30]$, and one of these included interviews among their cohort to support the quantitative data obtained from the PRO instrument [29]. Two longitudinal studies evaluated the impact of treatment on HRQoL of individuals with LTBI [18-25], one was a prospective cohort study identifying illness-related stigma among individuals with LTBI [20], one qualitative study exploring the experience of pregnant women with LTBI [23], and two retrospective studies evaluated the quality-adjusted life years (QALY) and mortality rates of individuals with LTBI [26-28]. The primary objectives of these studies varied, with four studies comparing the health and wellbeing of participants treated for LTBI and active TB disease [21, 22, 24-28], while one study compared the health and wellbeing of individuals treated for LTBI and individuals without TB infection [29]. Two studies compared the health and wellbeing of individuals treated for LTBI, active TB disease and individuals without TB infection [18, 19,30]. One cohort study [33] and one qualitative study [23] focused only on individuals with LTBI.

Five studies recruited participants from TB clinics [18, 19, 21-25, 27 28], one study recruited participants from the Infectious Disease or Paediatric Clinic [29], one study recruited participants from County Health Department [20], one study recruited participants from the primary care practice [30], and one study analysed data from the state-wide TB records and health authority registries [26].

\section{PRO instruments}

There was substantial heterogeneity in the instruments and methods used to assess health and wellbeing of individuals with LTBI among the included studies (table S3). Some PRO studies used generic measures to assess quality of life, such as the 36-item Short-Form Health Survey questionnaire (SF-36) and the EuroQol-5 (EQ-5D) tool [19, 22, 24, 25, 29, 30], while one study used a disease-specific quality of life measure, the St. George's Respiratory Questionnaire (SGRQ) [28]. Two studies examined the psychological wellbeing of their participants, using the Beck Depression Inventory (BDI) [24, 30] and Refugee Health Screener-15 (RHS-15) tool [29], while one study used the EMIC (Explanatory Model Interview Catalogue) for Tuberculosis in Haitian Populations questionnaire to develop a stigma scale for capturing illness-related stigma among individuals with LTBI [20]. The evaluation of psychometric properties of 


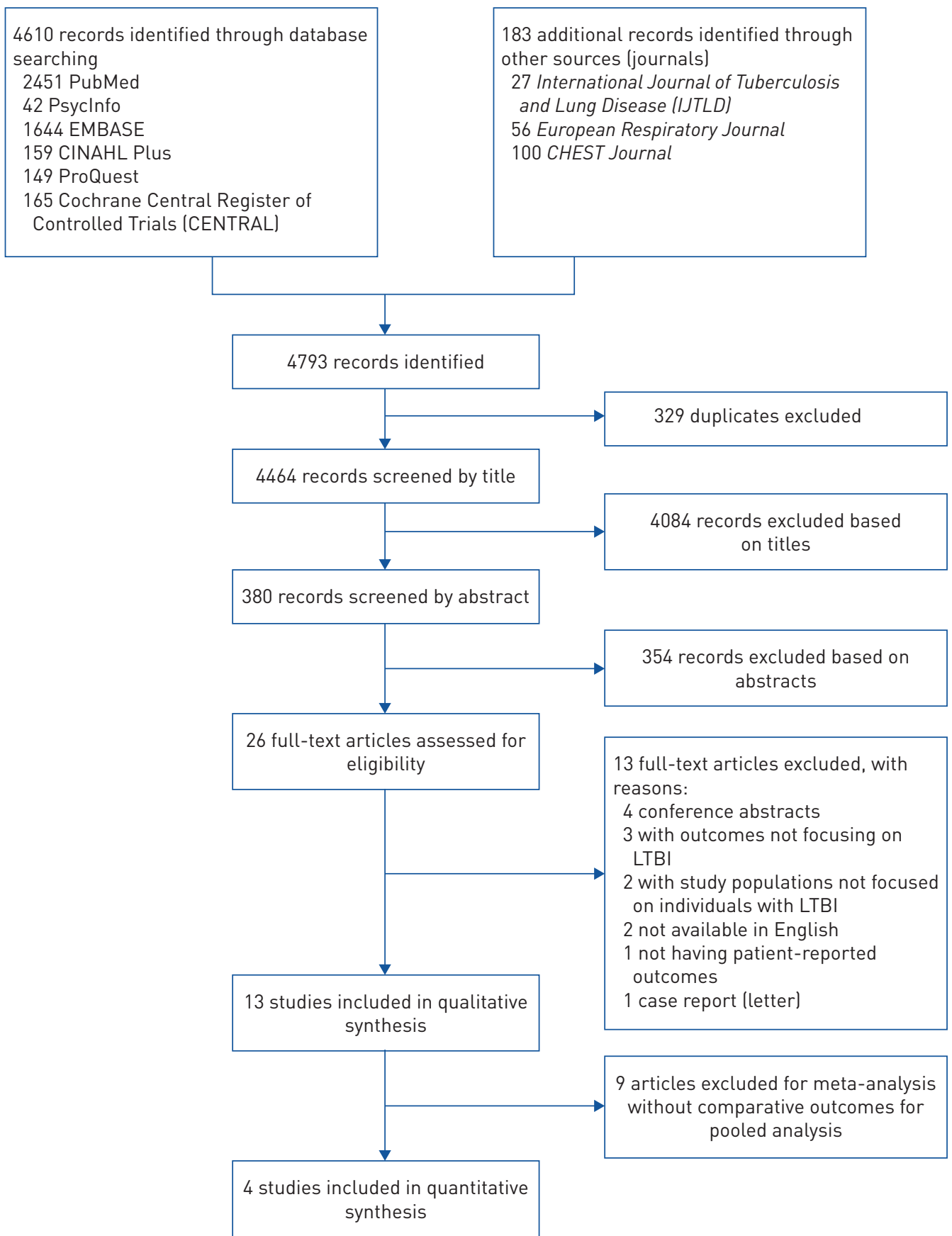

FIGURE 1 Flowchart showing the study selection process for the qualitative and quantitative synthesis. LTBI: latent tuberculosis infection.

HRQoL instruments in individuals with LTBI has not been demonstrated in any study. The reliability and validity of these HRQoL instruments and health utilities measures have only been tested in patients with active TB disease (table S4) $[18,19,21,22,25,30]$. As such, the results of HRQoL should be interpreted with caution as ceiling and floor effects were reported in several questionnaires used [18, 21, 22, 25, 28]. Publication bias and sensitivity analysis were not assessed as there were fewer than five studies available for the meta-analysis [34].

\section{Study quality}

The mean NOS quality score was 6.1 out of 9, indicating an overall fair quality of studies included in the current review (table S5). All eight studies had good quality in the selection of sample representativeness and comparability of cohorts. However, nearly all studies had not adequately reported the outcome 
measures [21, 22, 24-29]. This was because PRO for the quality of life and health utilities were collected through self-reported methods, which were at risk of recall bias affecting the validity and reliability of the results $[35,36]$. Two studies had recall periods of 1 and 4 weeks respectively as an attempt to reduce the risk of recall bias $[22,25]$. In two studies, there was a high rate of drop out $(>20 \%)[18,19,24]$. The impact of missing data was evaluated using multiple imputation in the study by BAUER and colleagues [18, 19], while the study by MARra and colleagues omitted the data from participants who were lost to follow-up [24].

The appraisal for the only qualitative study [23] fulfilled nine out of 10 items on the checklist, indicating that the methodological approaches used in designing, conducting and reporting the study were of high quality (table S6).

\section{Health and wellbeing}

In studies that reported HRQoL measured using the SF-36 questionnaire, the findings were summarised a mental component summary (MCS) and physical component summary (PCS) scores. These studies reported that LTBI had limited impact on the overall wellbeing of the diagnosed individuals, with comparable HRQoL to individuals without TB infection. There was some evidence to suggest that LTBI had some impact on psychosocial aspects such as stigmatisation and fear, which may have a greater impact than clinical symptoms especially among those who were not well-informed about LTBI [20, 23, 29, 30]. Pooled analysis of the two studies $[18,25]$ comparing health utilities between individuals diagnosed with LTBI and patients with active TB disease showed that individuals with LTBI had better health utilities than patients with active TB disease at baseline, with a mean difference (MD) of 0.13 (95\% CI 0.10 to 0.16 ; $\mathrm{p}<0.001$ ) (figure 2).

\section{Mental health and wellbeing}

The SF-36 questionnaire also provided an MCS score summarising scores from the following domains: vitality (VT), social functioning (SF), role emotional (RE) and mental health $(\mathrm{MH})$. Since the domains contributing to MCS represent both mental health (RE and MH) and social health (VT, SF) components; explanation on VT and SF will be explicitly explained in the social health and wellbeing subcategory.

The pooled MCS scores were higher in individuals with LTBI compared with patients with active TB disease (MD 4.44, 95\% CI -0.81 to $9.69, \mathrm{p}=0.10$ ) (figure S2). However, the finding was not statistically significant. Breaking down into the domains of SF-36, individuals with LTBI had higher domain scores for role emotion (MD 12.10, 95\% CI 3.21 to 20.99, $\mathrm{p}=0.008$ ) (figure S9).

When compared with individuals without TB infection, individuals with LTBI had lower MCS scores (MD $-2.35,95 \%$ CI -5.18 to $0.48, \mathrm{p}=0.10$ ) (figure S12), but this was not statistically significant. Breaking down into the domains of SF-36, the pooled estimate showed that individuals with LTBI had worse domain scores for the mental health domain compared with the individuals without TB infection (MD $-4.16,95 \%$ $\mathrm{CI}-7.45$ to $-0.87, \mathrm{p}=0.01$ ) (figure 3 ).

Moreover, two questionnaires were used to measure depression, including the single question from EQ-5D as well as the Beck Depression Index (BDI) [22, 24, 29, 30]. In all studies, there was suggestion that individuals with LTBI suffered from some form of depression, which was comparable to patients having active TB disease $[29,30]$. Interestingly, individuals with LTBI had reported a sense of fear and disbelief upon the diagnosis of the latent illness, with pregnant women interviewed in a qualitative study, having worries due to the misperception and a lack of knowledge about LTBI [23]. Individuals treated for LTBI were worried about TB re-activation, imposing undesirable stress to their life [29]. Additionally, the data from RHS-15 showed the evidence of mental distress among more than one-third of the immigrants diagnosed with LTBI [29].

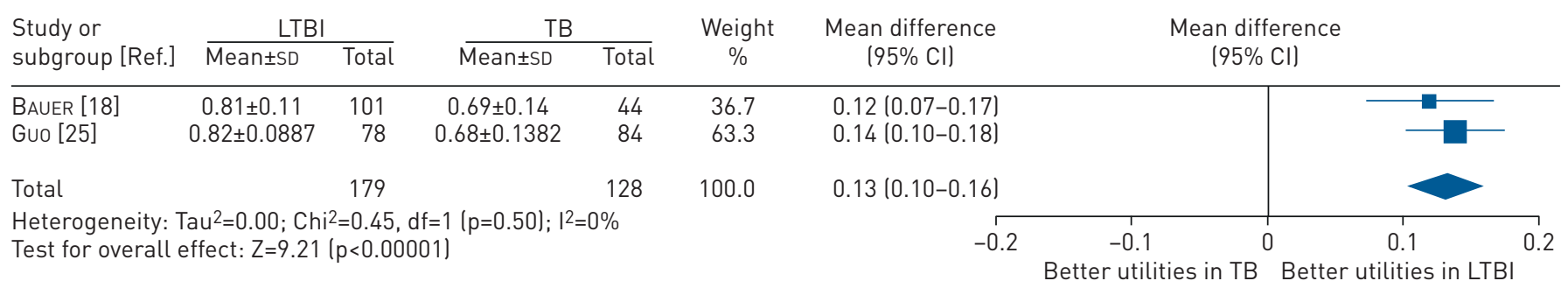

FIGURE 2 Mean difference (inverse variance method, random-effects model) between SF-6D utility scores for individuals treated for LTBI compared with patients treated for active TB disease. LTBI: latent tuberculosis infection; SF-6D: Short-Form Six-Dimension; TB: tuberculosis. 


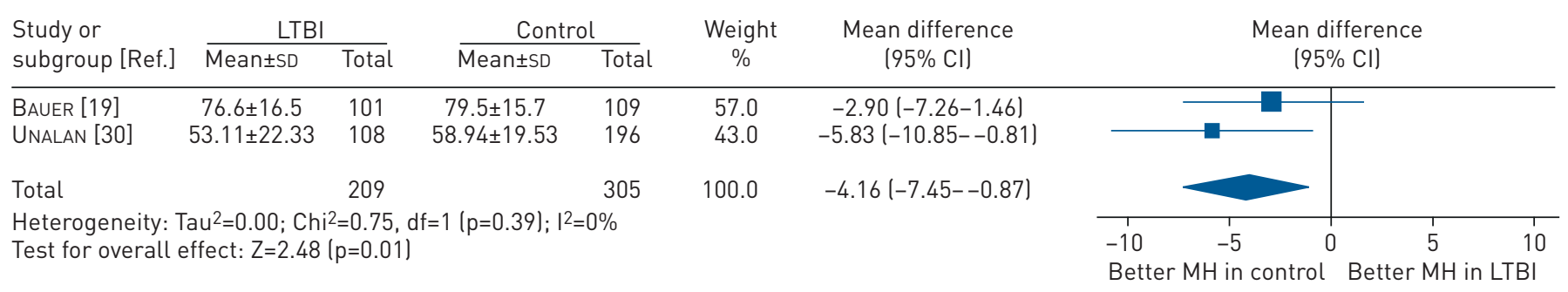

FIGURE 3 Mean difference (inverse variance method, random-effects model) between SF-36 mental health (MH) domain scores for individuals treated for LTBI compared with individuals without TB. LTBI: latent tuberculosis infection; SF-36: 36-item Short-Form Health Survey; TB: tuberculosis.

In a longitudinal study, the MCS score for individuals treated for LTBI was comparable before, during and after the treatment completion, but the domains of RE and $\mathrm{MH}$ deteriorated in the first month of treatment initiation, and increased as treatment continued. This suggests that individuals with LTBI may possibly experience mental health issues during treatment initiation [24].

\section{Physical health and wellbeing}

The PCS score from the SF-36 questionnaire summarises the scoring from domains including physical functioning (PF), role physical (RP), bodily pain (BP) and general health (GH).

The pooled PCS scores were higher in individuals with LTBI compared with patients with active TB disease (MD 4.36, 95\% CI 0.29 to $8.42, \mathrm{p}=0.04$ ) (figure 4). Individuals with LTBI had higher domain scores for PF (MD 12.96, 95\% CI 8.56 to 17.37, $\mathrm{p}<0.001$ ) (figure S3) and RP (MD 16.81, 95\% CI 6.95 to 26.68, $\mathrm{p}<0.001$ ) (figure $\mathrm{S} 4$ ).

However, when compared with individuals without TB infection, individuals with LTBI had lower PCS scores ( $\mathrm{MD}-2.40,95 \% \mathrm{CI}-7.50$ to $2.70, \mathrm{p}=0.36$ ) (figure $\mathrm{S} 11$ ), but did not reach statistical significance. Nevertheless, individuals with LTBI had poorer domain scores for BP (MD $-5.35,95 \%$ CI -9.59 to -1.12 , $\mathrm{p}=0.01$ ) (figure S15).

Since LTBI is an asymptomatic condition, the physical health of individuals with LTBI is preserved. However, pulmonary impairment was identified in one-fifth of the LTBI cohort, with reduced forced vital capacity (FVC) and/or forced exhalation volume $\left(\mathrm{FEV}_{1}\right)$ [28]. The finding from EQ-5D questionnaire from a study showed that one-quarter of individuals with LTBI experienced pain/discomfort, with three participants particularly relating the condition to the consumption of medication during treatment [29]. In addition, approximately $38 \%$ of individuals with LTBI from another study also reported incidence of intolerance to LTBI treatment within the first month of treatment initiation [24].

\section{Social health and wellbeing}

In term of the SF domain which also contributes to the MCS scores, individuals with LTBI had higher SF scores compared with patients with active TB disease (MD: 19.18; 95\% CI 5.33 to 33.03; p=0.007) (figure S8).

In a longitudinal study, the domains of VT and SF deteriorated in the first month of treatment initiation, and increased as treatment continued, indicating potential impact of LTBI treatment initiation on social health and wellbeing [24]. A qualitative study revealed the reluctance of pregnant women with LTBI in

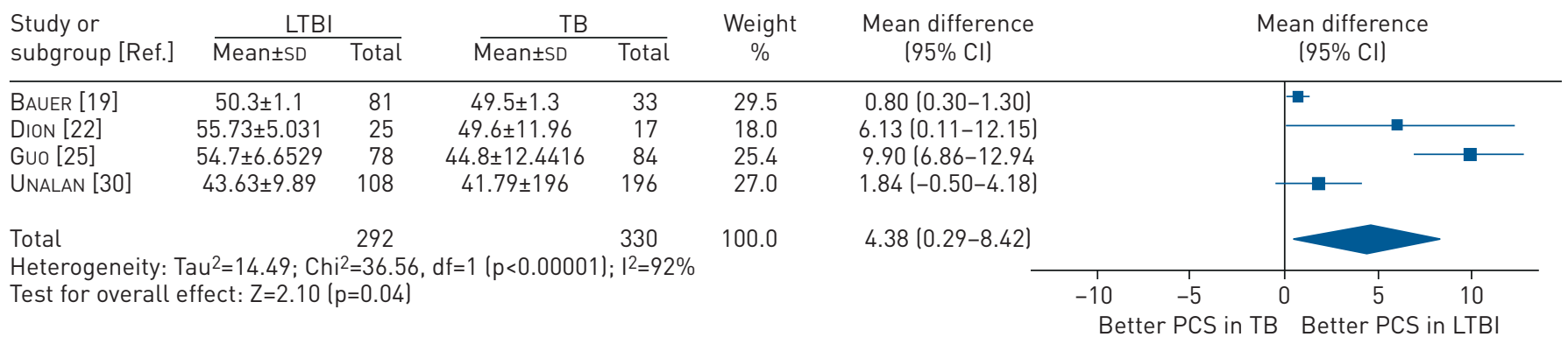


disclosing their status of diagnosis due to stigma [23]. One study highlighted the issue on stigma among defaulted participants who experienced distress, close contacts of patients with TB, participants who were not well-informed about LTBI and participants who were under close surveillance [20]. Moreover, another study had summarising remarks on the effect of stigma affecting the HRQoL among individuals with LTBI [29].

\section{Discussion}

PRO is an important component of public health surveillance and its assessment outcomes are increasingly being used as an indicator to address broader areas of public policy [15, 16, 37]. This is particularly important in conditions such as LTBI, where it can be used to obtain medical management feedback from affected individuals including issues related to treatment side effects, depression, distress or anxiety which may affect their quality of life and treatment completion rate $[15,16]$. This indirectly determines the burden of preventing TB re-activation and to guide the development of new strategic plans for future community-based interventions with quality integrated care among individuals with LTBI, helping towards achieving the WHO End TB Goal by 2035. As the first review focusing primarily on the health and wellbeing of individuals with LTBI, the findings from this study showed that individuals with LTBI had comparable HRQoL and health utilities with individuals without TB infection, but better than patients with active TB disease. The results from our review are in agreement with previous systematic reviews which indicated that active TB disease reported poorer HRQoL compared with individuals treated for LTBI $[2,3]$. Nevertheless, our understanding of the long-term impact of LTBI is considerably limited. While there have been several studies which have examined the impact of treatment on individuals with LTBI, only two studies have examined the changes in HRQoL and health utilities longitudinally over time in individuals treated for LTBI $[18,24]$.

Our review identified several areas where research focus can be further improved. In particular, we found that the mental wellbeing among individuals with LTBI has not been given sufficient attention. Only three studies had highlighted the mental health issues of depression, anxiety and fear encountered by individuals with LTBI [23, 29, 30]. Individuals with LTBI in these studies described the insecurities and misconceptions that LTBI was fatal and contagious attributed to the lack of knowledge and information on LTBI among communities. The findings were consistent with a previous systematic review which discussed about the fear and anxiety of having LTBI, deteriorating the mental wellbeing among individuals with LTBI [3]. In addition, the previous review also highlighted the lack of focus on psychological and social impact of LTBI among affected individuals, as they were also subjected to similar degrees of anxiety and fear of having the latent illness as patients having active TB disease [3].

In term of social wellbeing, two studies in this review identified the presence of stigma among individuals with LTBI that affected their quality of life and interrupted LTBI treatment $[20,29]$. A mixed-method study assessing the knowledge and perception of LTBI reported similar concerns on stigma and a lack of understanding on LTBI which may affect the acceptance on LTBI treatment plan among Chinese immigrants in Canada [12]. Furthermore, studies which investigated LTBI treatment barriers also raised the problems of fear and stigma among migrant participants upon positive screening for TST and IGRA $[10,38]$. With limited studies, the association between LTBI and social wellbeing, in particular stigma, has not been extensively understood in all levels of communities.

To date, several disease-specific HRQoL instruments have been developed and validated to measure PRO among patients with active TB disease in local communities, namely Quality of Life Instruments for Chronic Diseases, Functional Assessment of Chronic Illness Therapy - Tuberculosis and DR-12 score $[3,13,38,39,40]$. Similarly, multiple generic HRQoL tools have been adapted for patients with active TB disease, including the Duke Health Profile, the WHO Quality of Life-BREF, and the Medical Outcome Study, but none have been used in individuals with LTBI $[3,13,41-43]$. This review found that only three tools (SF-36, EQ-5D and SGRQ) have been used to measure HRQoL outcomes in individuals with LTBI $[19,22,24,25,28-30]$ and while these have been used in other TB studies, LTBI-specific outcome measure are lacking.

The SF-36 tool was the most commonly used instrument to measure HRQoL in LTBI $[19,22,24,25,30]$. Although it appears to be a valid and reliable tool in active TB disease, this cannot be similarly inferred for LTBI. As shown in this study, LTBI impacts various domains of a person's life, including the mental, social as well as quality of life. However, the current tools available could only capture parts of the health domains in individuals with LTBI; thus the outcome measures may not be sufficiently sensitive to observe the actual impact on HRQoL. Since LTBI is asymptomatic and noninfectious without any physical sign of debility, the possible impact of the latent illness on mental and psychosocial wellbeing may have been overlooked. Despite individuals with LTBI not being affected by clinical symptoms of active TB disease, physical wellness could still be an important factor to capture in individuals with LTBI in order to detect possible episodes of TB re-activation. 
Since HRQoL is still an infrequently explored area in LTBI research, and there is a need for a LTBI-specific PRO measure which could effectively capture all health aspects in a person with LTBI including the association of HRQoL with physical health, mental health, stigma and treatment-related adverse events. Indeed, studies have shown that these self-assessed health status tools can be more powerful predictors of morbidity and mortality than many other objective measures currently in use, and would be useful to identify and deliver health care needs through policy improvement $[44,45]$.

Extensive research will be required to determine the impact of the psychological, emotional and social determinants of the latent illness among individuals with LTBI particularly in countries with intermediate and high TB burdens. This is to ensure that sufficient resources are allocated to incorporate psychosocial support and interventions together with efficient LTBI information dissemination to local communities as well as continuous training for health care workers to assist individuals with LTBI to safeguarding the health and wellbeing of individuals with LTBI $[46,47]$.

The findings from this review further support the evidence of advocating for TB preventive care with early LTBI diagnosis and treatment to avoid more severe health and wellbeing repercussions, together with the socioeconomic burden as a consequence of active TB disease [48]. Pulmonary impairment may persist even after treatment completion and clinical recovery with the risk of post-tuberculosis chronic illness and respiratory disease $[28,48]$. Hence, concerted efforts will be required to encourage high-risk communities to screen for TB. Early detection and treatment for individuals with LTBI would be beneficial in preventing detrimental consequences of active TB disease. This will need to go hand-in-hand with some form of social protection mechanisms to ensure that individuals with LTBI do not undergo financial hardships while being on treatment.

In this review, we found limited studies on health and wellbeing among individuals with LTBI, and no studies from high TB burden countries. Since LTBI is asymptomatic, the importance of health and wellbeing in individuals with LTBI may not be acknowledged, particularly in countries with a high TB burden where the principal focus is to tackle active TB disease. Potential research on health and wellbeing among individuals with LTBI would be useful where cost-utility analysis can be conducted using health utilities data, providing evidence for policy decisions for the implementation of LTBI management in lowand middle-income countries (LMICs). Moreover, psychosocial barriers among individuals with LTBI could be identified and overcome to achieve the desired health outcomes in LTBI management in addition to the effort to eliminate TB in accordance with WHO's End TB Strategy as well as achieving Sustainable Development Goal (SDG) 3.3.2 [8]. This highlights the importance of health education and promotion in LTBI management, to improve the health outcomes of patients with TB. Future studies should also incorporate social values as part of the holistic medical service, as we strive towards the status of countries with low TB burdens by $2035[8,49]$.

Nevertheless, this review has several limitations. Firstly, there was substantial heterogeneity in the included studies, in terms of design and aims. The psychometric properties of the instruments used for measuring HRQoL in LTBI have not been extensively examined. Ceiling and floor effects were reported in eight of the tools used $[18,21,22,25,28]$, suggesting that the results should be interpreted with caution as these tools appear to be unable to measure differences in HRQoL between individuals with LTBI and individuals without TB infection. This may also help explain the lack of difference in HRQoL observed in the identified studies, despite results from the qualitative studies which had reported otherwise. Finally, our review only included published research, and thus we may have missed some relevant studies. Nonetheless, we believe that this is minimal given our broad inclusion criteria, with searches in six databases supplemented with a search of grey literature to account for publication bias.

\section{Conclusion}

The review suggests that while the HRQoL of individuals with LTBI was minimally affected, these individuals experienced some form of mental health impairment, especially anxiety and fear. In such circumstances, as HRQoL addresses broader scopes of health beyond the direct measures of disease outcome; it could be a more sensitive approach to reflect the existence of additional wellbeing challenges in LTBI management. The recognition of potential psychological and social barriers as well as the effect of stigma in LTBI management is beneficial in guiding policy improvements for better quality of care for patients with LTBI. This will help reduce the global TB burden, and move towards achieving WHO's End TB Strategy target and the United Nations' 2030 SDGs.

Acknowledgements: The authors would like to thank the Director General of Health, Ministry of Health, Malaysia for his permission to publish this article.

Conflict of interest: None declared. 


\section{References}

1 World Health Organization. Tuberculosis Key Facts. Geneva, 2020. Available from: www.who.int/news-room/ fact-sheets/detail/tuberculosis Date last accessed August 7, 2020.

2 Bauer M, Leavens A, Schwartzman K. A systematic review and meta-analysis of the impact of tuberculosis on health-related quality of life. Qual Life Res 2013; 22: 2213-2235.

3 Guo N, Marra F, Marra CA. Measuring health-related quality of life in tuberculosis: a systematic review. Health Qual Life Outcomes 2009; 7: 14-14.

4 Sweetland AC, Kritski A, Oquendo MA, et al. Addressing the tuberculosis-depression syndemic to end the tuberculosis epidemic. Int J Tuberc Lung Dis 2017; 21: 852-861.

5 Tanimura T, Jaramillo E, Weil D, et al. Financial burden for tuberculosis patients in low- and middle-income countries: a systematic review. Eur Respir J 2014; 43: 1763-1775.

6 United Nations. Political Declaration of the UN General Assembly High Level Meeting. New York, 2018. www. who.int/tb/unhlmonTBDeclaration.pdf Date last accessed August 7, 2020.

7 World Health Organization. Tuberculosis (TB) - Latent Tuberculosis Infection (LTBI)- FAQs. Geneva, 2015 www. who.int/tb/areas-of-work/preventive-care/ltbi/faqs/en/ Date last accessed August 7, 2020.

8 World Health Organization. The End TB Strategy. Geneva, 2018. www.who.int/tb/post2015_TBstrategy.pdf?ua=1 Date last accessed August 7, 2020.

9 Brown J, Capocci S, Smith C, et al. Health status and quality of life in tuberculosis. Int J Infect Dis 2015; 32: 68-75.

10 Fox GJ, Dobler CC, Marais BJ, et al. Preventive therapy for latent tuberculosis infection - the promise and the challenges. Int J Infect Dis 2017; 56: 68-76.

11 Gao J, Berry NS, Taylor D, et al. Knowledge and perceptions of latent tuberculosis infection among Chinese immigrants in a canadian urban centre. Int J Family Med 2015; 2015 : 546042.

12 Mason PH, Degeling C, Denholm J. Sociocultural dimensions of tuberculosis: an overview of key concepts. Int J Tuberc Lung Dis 2015; 19: 1135-1143.

13 Khan S, Tangiisuran B, Imtiaz A, et al. Health status and quality of life in tuberculosis: systematic review of study design, instruments, measuring properties and outcomes. Health Sci J 2017; 11: 1.

14 Kastien-Hilka T, Abulfathi A, Rosenkranz B, et al. Health-related quality of life and its association with medication adherence in active pulmonary tuberculosis - a systematic review of global literature with focus on South Africa. Health Qual Life Outcomes 2016; 14: 42.

15 van der Wees PJ, Verkerk EW, Verbiest MEA, et al. Development of a framework with tools to support the selection and implementation of patient-reported outcome measures. J Patient Rep Outcomes 2019; 3: 75.

16 Rivera SC, Kyte DG, Aiyegbusi OL, et al. The impact of patient-reported outcome (PRO) data from clinical trials: a systematic review and critical analysis. Health Qual Life Outcomes 2019; 17: 156.

17 Stroup DF, Berlin JA, Morton SC, et al. Meta-analysis of observational studies in epidemiology: a proposal for reporting. Meta-analysis Of Observational Studies in Epidemiology (MOOSE) group. JAMA 2000; 283: 2008-2012.

18 Bauer M, Ahmed S, Benedetti A, et al. The impact of tuberculosis on health utility: a longitudinal cohort study. Qual Life Res 2015; 24: 1337-1349.

19 Bauer M, Ahmed S, Benedetti A, et al. Health-related quality of life and tuberculosis: a longitudinal cohort study. Health Qual Life Outcomes 2015; 13: 65.

20 Coreil J, Lauzardo M, Clayton H. Stigma and Therapy completion for latent tuberculosis among Haitian-origin patients. Fla Public Health Rev 2010; 7: 32-38.

21 Dion M, Tousignant P, Bourbeau J, et al. Measurement of health preferences among patients with tuberculous infection and disease. Med Deci Making 2002; 22: Suppl. 5, S102-S114.

22 Dion M, Tousignant P, Bourbeau J, et al. Feasibility and reliability of health-related quality of life measurements among tuberculosis patients. Qual Life Res 2004; 13: 653-665.

23 Jansson L, Shedrawy J, Lönnroth K, et al. Latent tuberculosis in pregnant women: a patient perspective. Int $J$ Tuberc Lung Dis 2020; 24: 310-315.

24 Marra CA, Marra F, Colley L, et al. Health-related quality of life trajectories among adults with tuberculosis: differences between latent and active infection. Chest 2008; 133: 396-403.

25 Guo N, Marra C, Marra F, et al. Health state utilities in latent and active tuberculosis. Value Health 2008; 11: $1154-1161$.

26 Miller TL, Wilson FA, Pang JW, et al. Mortality hazard and survival after tuberculosis treatment. Am J Public Health 2015; 105: 930-937.

27 Miller TL, McNabb SJN, Hilsenrath P, et al. Personal and societal health quality lost to tuberculosis. PLoS One 2009; 4: e5080

28 Pasipanodya JG, Miller TL, Vecino M, et al. Using the St. George Respiratory Questionnaire to ascertain health quality in persons with treated pulmonary tuberculosis. Chest 2007; 132: 1591-1598.

29 Shedrawy J, Jansson L, Röhl I, et al. Quality of life of patients on treatment for latent tuberculosis infection: a mixed-method study in Stockholm, Sweden. Health Qual Life Outcomes 2019; 17: 158.

30 Unalan D, Soyuer F, Ceyhan O, et al. Is the quality of life different in patients with active and inactive tuberculosis? Int J Infect Dis 2008; 55: 127-137.

31 Wells G, Shea B, O'Connell D, et al. The Newcastle-Ottawa Scale (NOS) for assessing the quality of nonrandomised studies in meta-analyses. 2011. www.ohri.ca/programs/clinical_epidemiology/oxford.asp Date last accessed August 7, 2020.

32 Critical Appraisal Skills Programme. Qualitative Checklist. 2018. www.casp-uk.net/wp-content/uploads/2018/01/ CASP-Qualitative-Checklist-2018.pdf Date last accessed August 7, 2020.

33 Wan X, Wang W, Liu J, et al. Estimating the sample mean and standard deviation from the sample size, median, range and/or interquartile range. BMC Med Res Methodol 2014; 14: 135.

34 Higgins JPT, Thomas J, Chandler J, et al. Cochrane Handbook for Systematic Reviews of Interventions, version 6.0 [updated July 2019]. Cochrane, 2019.

35 Althubaiti A. Information bias in health research: definition, pitfalls, and adjustment methods. J Multidiscip Healthc 2016; 9: 211-217. 
Dowling NM, Bolt DM, Deng S, et al. Measurement and control of bias in patient reported outcomes using multidimensional item response theory. BMC Med Res Methodol 2016; 16: 63.

37 Kindig DA, Booske BC, Remington PL. Mobilizing Action Toward Community Health (MATCH): metrics, incentives, and partnerships for population health. Prev Chronic Dis 2010; 7: A68.

38 Liu Y, Birch S, Newbold KB, et al. Barriers to treatment adherence for individuals with latent tuberculosis infection: a systematic search and narrative synthesis of the literature. Int J Health Plann Manage 2018; 33: e416-e433.

39 Sun Y, Yang Z, Wan C, et al. Development and validation of the pulmonary tuberculosis scale of the system of Quality of Life Instruments for Chronic Diseases (QLICD-PT). Health Qual Life Outcomes 2018; 16 : 137.

40 Dhingra VK, Rajpal S. Health related quality of life (HRQL) scoring (DR-12 score) in tuberculosis - additional evaluative tool under DOTS. J Commun Dis 2005; 37: 261-268.

41 Masumoto S, Yamamoto T, Ohkado A, et al. Factors associated with health-related quality of life among pulmonary tuberculosis patients in Manila, the Philippines. Qual Life Res 2014; 23: 1523-1533.

42 Chung WS, Lan YL, Yang MC. Psychometric testing of the short version of the World Health Organization quality of life (WHOQOL-BREF) questionnaire among pulmonary tuberculosis patients in Taiwan. BMC Public Health 2012; 12: 630.

43 Duyan V, Kurt B, Aktas Z, et al. Relationship between quality of life and characteristics of patients hospitalised with tuberculosis. Int J Tuberc Lung Dis 2005; 9: 1361-1366.

44 DeSalvo KB, Bloser N, Reynolds K, et al. Mortality prediction with a single general self-rated health question. A meta-analysis. J Gen Intern Med 2006; 21: 267-275.

45 Dominick KL, Ahern FM, Gold CH, et al. Relationship of health-related quality of life to health care utilization and mortality among older adults. Aging Clin Exp Res 2002; 14: 499-508.

46 Misselbrook D. W is for wellbeing and the WHO definition of health. Br J Gen Pract 2014; 64: 582-582.

47 World Health Organization. Preamble to the Constitution of the World Health Organization as adopted by the International Health Conference, New York, 19-22 June 1946 and entered into force on 7 April 1948. 1948. www. who.int/about/who-we-are/constitution Date last accessed August 7, 2020.

48 Basham CA, Romanowski K, Johnston JC. Life after tuberculosis: planning for health. Lancet Respir Med 2019; 7: 1004-1006.

49 Kruk ME, Gage AD, Arsenault C, et al. High-quality health systems in the Sustainable Development Goals era: time for a revolution. Lancet Glob Health 2018; 6: e1196-e1252. 